\title{
Exam Conduction and Proctoring System Using Face Detection
}

Prof. Hemant B. Shinde BRACT VIT, Pune, India

\author{
Rohan Ranjan 11910134 \\ Dept.Of Computer Engineering \\ BRACT VIT, Pune, India
}

\author{
Varun Ringnekar 11910590 \\ Dept.Of Computer Engineering \\ BRACT VIT, Pune, India
}

\author{
Himangi Rinwa - 11910386 \\ Dept.Of Computer Engineering, \\ BRACT VIT, Pune, India
}

\author{
Rohit Singh - 11910771 \\ Dept. Of Computer Engineering, \\ BRACT VIT, Pune, India
}

\section{Abstract}

The Online Examination Portal is a web application for taking an online test productively along with face recognition capabilities to perform live proctoring, and there is no time wasted for checking the paper. This report will incorporate all highlights and procedures which are required to develop this portal. This document incorporates details about the objective of the system, approximately targets of the system, system scope confinement, essential system requirements, group advancement, likely venture risks, schedule of the deployment, and finally observing and reporting mechanisms for the whole system. Online Examination Conducting Portal is exceptionally useful for Instructive Institute's to prepare a complete exam, conduct proctoring to prevent misconduct, secure the time that will take to check the paper, and plan check sheets. Online Examination Portal will help the Institutes to test understudies and develop their abilities. But the impediments for the Online Exam systems, it takes more time when the user prepares the exam at the primary time for utilization. To conduct the exam we require the number of computers with the same number of students. With the successful use of the Examination Portal, the facilitator can utilize this system to create the tests as their requirements and we can get accurate results and save time once deployed.

Keywords: Online Examination.

\section{INTRODUCTION}

Nowadays, Online Examination Framework is considered a quick creating examination strategy since of its exactness and speed. It is additionally required less labor to handle the examination. Nearly all organizations nowadays are managing their exams by an online examination system, since it decreases student's time in examinations.

Institutes can moreover effectively monitor the advancement of the student that they deliver through an examination. The question sets will bolster the automated preparation and it is a critical part in a random era of unique question sets of address papers. The most extreme number of questions is better randomization. As a result of this, the result is calculated in less time. It moreover helps to decrease the need for paper. All the entrance exams are held online because of their quick and precise marking scheme. Online examination is more adaptable than written examination. It is essentially a plan to promote variety in the education system. Due to online examinations, there's less chance of losing the judgment of exam designs. E.g. it is 
the slightest conceivable for arranging the online examination set up when compared with another examination system.

The online examination system created based on Java web can not as it was made full utilize of different resources on the campus but to make full use of the different educating resources of the school. The use of instructing resources also crosses the limitations of time and space through the arrangement. Instructors and examinees give helpful and fast learning devices, which can share instructing assets on a bigger scale and can improve education proficiency and realize education. To change conventional examination strategies, set up a reasonable examination mechanism, and realize instruction and education in a logical and standardized way. Administration, to play down the overwhelming work of teachers, such as planning questions, composing, and judging papers. Realize that candidates can take the test online and review the test in time. The instructor gets the examinee's examination circumstance, conducts a sensible investigation, and makes strides in the quality of education.

The portal has two login options, one for admin and another for students. Admin has the privilege of adding a test, scheduling a test, and adding a student. The student side has a dashboard on which they can see their upcoming tests and check their scores on the previous tests and look at the tests that they have missed. Once the test time approaches the start button on the test becomes active and then the student is taken to the test page where our algorithm selects questions that are assigned for the test and displays them to the student and saves the answers. The student is asked for the camera access without which the exam won't start and a face detection api is run to make sure that there is no misconduct being carried out. Once the student submits the test the score gets calculated and updated on the database

\section{OBJECTIVES}

Online Examination may be a total end-to-end arrangement to cover all aspects of the online examination system. The fundamental objective of creating this project is: Provides total site arrangement, counting part enrollment, giving tests, storing of results. Complete web-based administration. The online examination system can naturally add the marks allocated in each address to decide the overall stamp for the test. The system will be able to calculate the examination marks and display the result quickly after completing the exam. students must view the right reply to the address after completing the test. The system has effective coherent get to administration input, each user must be distinguished by utilizing an id and a password confirmation approach is connected to secure the examination system. The Online Examination system produces several reports for administration like Add up to students/members enrolled for the examination, no of available examinations, tests given by the understudy.

\section{Literature Survey}

Zhenming et al(2003): Developed an online examination system based on a web browser/server framework, that supports some basic features, and carries out the examination, and provides the auto-grading system for objective questions and operating questions like programming, MS Word, Powerpoint, MS windows, Excel, etc. SIETTE Guzman and Cenejo (2005): They developed an online examination system called SIETE (System of intelligent Evaluation using Tests for Tele Education). This system provides login and some basic features but lacks the premium features such as random questions selection and random choices distribution, resumption capabilities, random questions distribution.

Ayo et al (2007): They proposed a model of Online Examination. The software was 
developed in a private university in Nigeria. This software was developed to conduct the entrance examination for all Nigerian universities called JAMB(Joint Admission Matriculation Board). This software was designed and tested at Covenant University, a private university in Nigeria. They found this software really helpful for conducting neat and clean entrance examinations. It eliminates the problems associated with the traditional methods of entrance examination.

\section{Proposed System}

This Online Examination portal comes up with a very unique design.

It has a navigation bar, which provides the user with many options like Home (gets user directly to the Home page of the site), About (provides information about the site), Our Team (introduces peers with Our team) \&, Contact Us (for contracting directly with the site owner to report any bug they faced or for pursuing any help) .

Admin module:

For an admin to login into the system, Admin doesn't have to provide any user id, he/she only has to put the right password as there is only one single admin login.

[i ] Creating a new test :

Admin has the privilege of adding and scheduling a test and adding a new student to the portal.

While adding a test, Admin has to first specify the department for which he is willing to create the test. For every question in the test, he/she will have to put four options and also specify the correct option out of four at the end. Admin also has the privilege of adding questions to an existing test by simply specifying the test name and the department for which the test was created.

\section{[ii ] Scheduling an existing test:}

For scheduling an existing test, Admin only has to put the name of the test and the department for which he is willing to schedule the test along with the date, time, and duration for the test.

If, in case an admin accidentally tries to schedule the test which does not exist then the portal has the feature to warn the admin that he is trying to schedule an unexisting test.

[iii] Registering a new student to the portal:

The registration process for new students is very simple as the admin only has to specify the name, department, id of the student and assign a password to secure the student's login.

Student module :

For a student to login into the portal, he/she will have to specify their correct student ID and password associated with it.

[ i ] Student Dashboard:

After the student has successfully logged in into the system by putting in valid credentials then he/she will be able to see his/her dashboard which will display all the scheduled and expired tests which are assigned to his/her department.

In the expired test section, the student will also be able to see the marks scored for already attempted tests. 
[ ii ]Test Page :

Once the student clicks on the start test, he/she will be directed to the test page. Here they will be asked for permission to access their camera. Once access is provided the test will start and their camera will be displayed at the bottom left of the screen without obscuring the test.

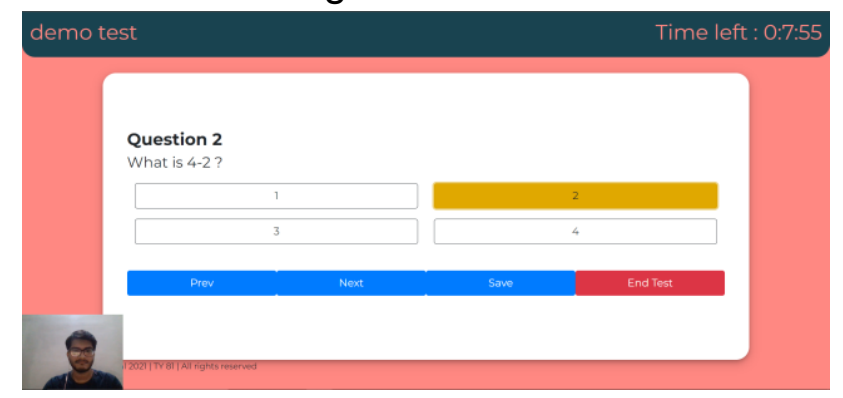

If a face is detected in the camera the test will run as intended however once the face stops being detected an alert would pop up warning the student to make sure that their face is visible and that if it is not visible in the next 45 seconds the exam will be automatically submitted.

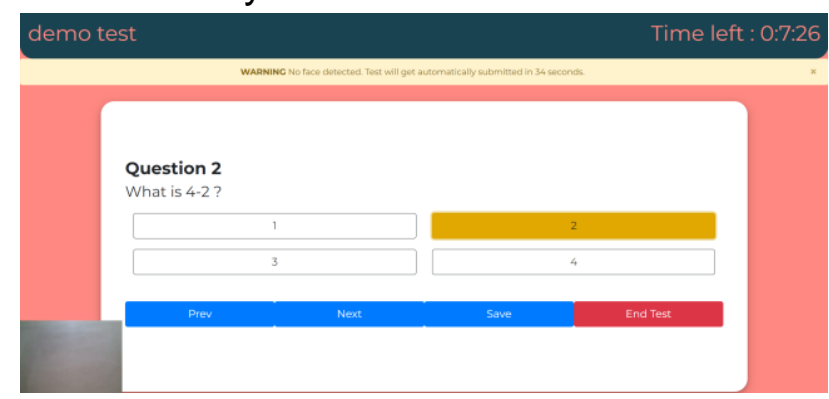

This is also the page where all the questions will be displayed.

Students can hover through the questions through the Prev and Next button.

Students can save the question through the Save button and proceed to the next question.

This page also has a timer at the top right corner which will keep on updating the student about time remaining for the test to end.
Once Clicked on End Test, the test will be submitted and Dashboard will be updated based on the marks obtained.

\section{PROJECT METHODOLOGY}

\section{Front End:}

This project uses HTML (Hyper-Text Markup Language), CSS (Cascading Style Sheets), and JavaScript for the front end.

In our project HTML has been used along with Bootstrap to build the basic structure of the website and to make the components dynamic

The bootstrap components used are:

\section{Container:}

Containers are the most basic layout element in Bootstrap and are required when using the default grid system.

We Used .container-fluid for a full width container, spanning the entire width of the viewport.

\section{Navbar:}

Navigation header and the navbar. Includes support for branding, navigation, and more, including support for the collapse plugin.

Navbars come with built-in support for a handful of sub-components. Choose from the following as needed:

- navbar-brand for your company, product, or project name.

- navbar-nav for a full-height and lightweight navigation (including support for dropdowns).

- navbar-toggler for use with our collapse plugin and other navigation toggling behaviors. 
- form-inline for any form controls and actions.

- .navbar-text for adding vertically centered strings of text.

- collapse.navbar-collapse for grouping and hiding navbar contents by a parent breakpoint.

This navbar is dynamic and based on the screen aspect ratio, it places the components inside to provide a fluid user interface.

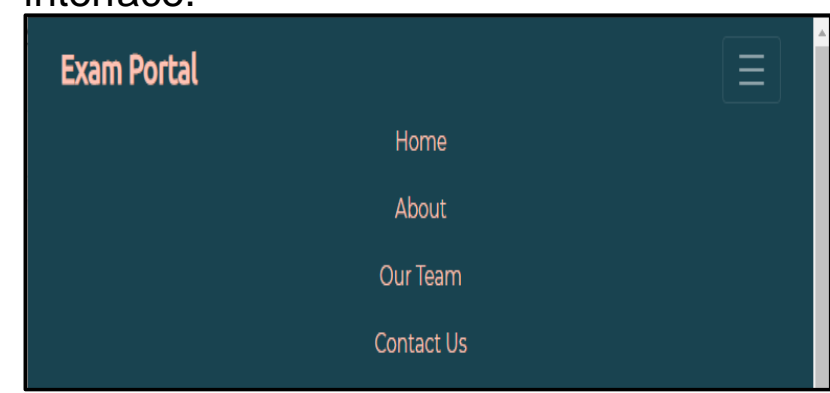

Form Groups:

In our project we have used the form groups to create our login page, student entry page, question entry page, test schedule page and the contact us page. The form groups contain components which allow us to take inputs from users, this includes components like:

- Email

- Password

- Checkbox

- RadioGroups

Student Login

Student ID

Enter your student ID

Password

Password

Submit

\section{Buttons:}

Bootstrap provides us with a huge range of customisable buttons with beautiful animations that are ready to use by just adding a class to your html <button> element

\section{Layout Classes:}

Bootstrap provides us with various layout classes which when applied to our HTML elements update their CSS code and positions them dynamically for devices with different aspect ratios

The different layout classes we have used are:

row: This class when added to a <div> converts that div into a flexbox which can dynamically change the position of the components inside of it

col: This class is added to a component inside the <div> which has the row class added to it and based on the type of class added the row flexbox allows the specified amount of blocks to that component out of twelve

In our project, we have used these classes to position our buttons on the test page so that they stack when they are placed in a smaller screen

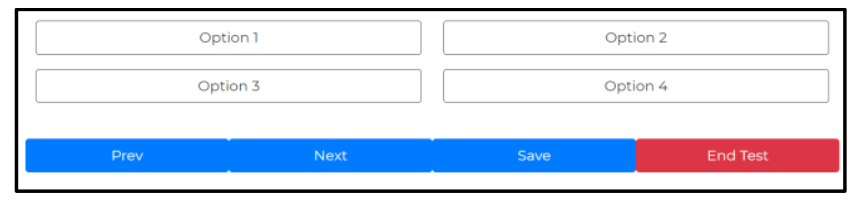




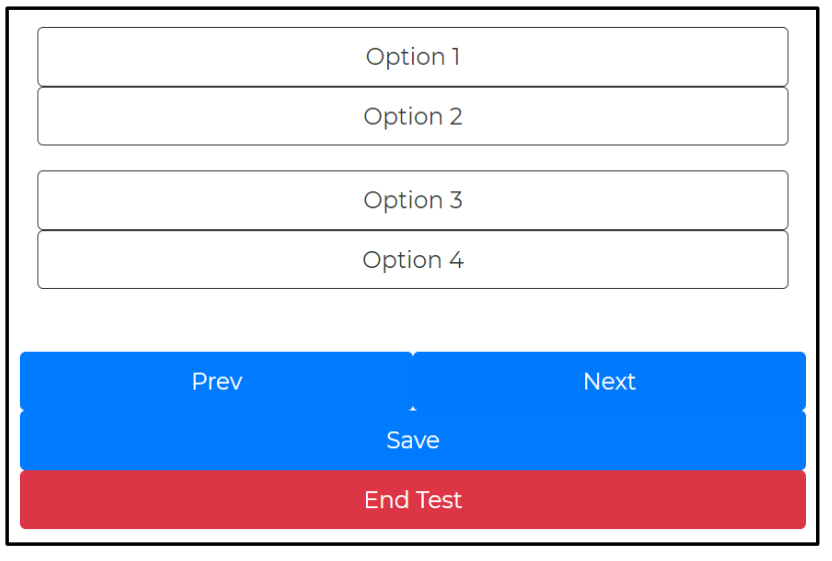

Back End:

This project uses javascript with Node.js and Express.js for serving files and providing a solid backend.

In our project we have used Node.js as the backend because of its speed and asynchronous property.

We have used Express.js because it is very easy to use and provides excellent routing. In our project Express.js provides routing for different urls and also provides a middleware for accessing the data passed through various GET and POST requests.

We have used mongoose framework to connect Node.js backend with the MongoDB database.

\section{Face API:}

For face detection, this project implements a SSD (Single Shot Multibox Detector) based on MobileNetV1. The neural network will compute the locations of each face in an image and will return the bounding boxes together with it's probability for each face. This face detector is aiming towards obtaining high accuracy in detecting face bounding boxes instead of low inference time. This data returned by the face-api is used to determine the presence of the student attempting the test.

The size of the quantized model is about 5.4
MB (ssd_mobilenetv1_model).

The face detection model has been trained on the WIDERFACE dataset

\section{Database:}

MongoDB is a document database built on a scale-out architecture that has become popular with developers of all kinds who are building scalable applications using agile methodologies.

In this project MongoDB has been used because the document data model is a powerful way to store and retrieve data that allows developers to move fast and also its scale-out architecture can support huge volumes of both data and traffic.

\section{CONCLUSION}

We have successfully created \& deployed an online examination portal. The portal

\section{REFERENCES}

[1] A web services-based online training and exam system(Hua Pang, Shu Yang, Liying Bian)Published in Wireless Communications, Networking and Mobile Computing, 2008. WiCOM '08. 4th International.

[2] Design and implementation of an online self-training system for the Computer System Platform course. Published in: Advanced Computational Intelligence (ICACl), 2012 IEEE Fifth International Conference on. 
[3]Tallent -

Runnels, M. K., et al. (2006). "Teaching

courses online: A

review of the research." Review of

educational research 76(1): 93

[4]Downing, D., et al. (2000). Dictionary of computer and Internet

terms, Barron's Educational series

[5] Fagbola Temitayo M., Adigun Adebisi A., Oke Alice O(2013)," Computer-Based Test (Cbt) System for University Academic Enterprise Examination", International Journal of Scientific \& Technology Research Volume 2, Issue 8, August 2013.

[6] I.M.M. Emary El and J.A.A. Abu (2006), "An Online Website for Tutoring and EExamination of Economic Course", American Journal of Applied Sciences 3 (2): Page 1715-1718, ISSN 1546-9239.

[7] O.A. Agbaji, O.T.Ruth and M.B. Sroyewun (2010), "Development of an EAssessment Platform forNigerian Universities", Research Journal Applied Sciences, Engineering and Technology 2(2): Page 170- 175, ISSN: 2040-7467. 